\title{
MULTIMODAL RECORDING OF BRAIN ACTIVITY IN HEATLHY TERM NEONATES DURING PHOTIC STIMULATION BY NEAR-INFRARED IMAGING AND ELECTROENCEPHALOGRAPHY
}

\author{
M. Biallas ${ }^{1,2}$, I. Trajkovic ${ }^{1,2}$, C. Hagmann ${ }^{1}$, F. Scholkmann ${ }^{1}$, L. Holper ${ }^{1,3}$, A. Beck $^{1,4}$, C. Jenny ${ }^{1}$, M. Wolf ${ }^{1}$
}

${ }^{1}$ Division of Neonatology, University Hospital Zurich, ${ }^{2}$ Institute for Biomedical Engineering, ${ }^{3}$ Institute of Neuroinformatics, University of Zurich and ETH Zurich, Zurich, ${ }^{4}$ Core Program of Structural Biology and Biophysics, Biozentrum, University of Basel, Basel, Switzerland

Background and aims: Is functional near-infrared imaging (NIRI) reliable enough to be clinically useful? To answer this question we studied functional brain activation (hemodynamic response by neurovascular coupling and optical neuronal signal) in neonates in a multimodal set-up, i.e. simultaneously recording NIRI and electroencephalography (EEG).

The aims of the study were:

(i) to determine the sensitivity and

(ii) repeatability of NIRI to detect the hemodynamic response,

(iii) the sensitivity and

(iv) repeatability of EEG to detect a visual evoked potential (VEP),

(v) to analyse optical data for the optical neuronal signal and

(vi) to test whether inadequate stimulation could be reason for absent hemodynamic responses.

Methods: 14 healthy term neonates (postnatal mean age 2.1 days) underwent photic flash stimulation during sleep on two different days. NIRI and EEG were acquired simultaneously.

\section{Results:}

(i) Sensitivity of NIRI was $61.5 \%$ to detect hemodynamic responses;

(ii) their repeatability was $41.7 \%$. A VEP was detected

(iii) in $96.3 \%$ of all subjects with

(iv) a repeatability of $92.3 \%$.

(v) In two measurements, data met the criteria for an optical neuronal signal.

(vi) By comparison with EEG data, insufficient stimulation was excluded as reason for absent hemodynamic responses.

Conclusions: NIRI is a promising tool to study cognitive activation and development of the brain. For clinical application, however, the sensitivity and repeatability on an individual level still needs to be improved. EEG in contrast has an adequate sensitivity and repeatability. 